\title{
PARALISIA CEREBRAL: FUNDAMENTOS PARA PAIS E CUIDADORES
}

\author{
CEREBRAL PALSY: BASICS FOR PARENTS AND CAREGIVERS
}

*Carlos Bandeira de Mello Monteiro, **Talita Dias da Silva

\section{RESUMO}

Um aspecto que não pode ser ignorado é o impacto da paralisia cerebral na dinâmica interna da família, devido ao fato de que seus membros são confrontados com uma realidade diferente que exige adaptações e modificações diárias. A forma como os pais, cuidadores e membros da família se ajustam é crucial para o futuro da criança e das pessoas envolvidas. Devido às dificuldades dos pais e cuidadores de crianças com paralisia cerebral em adquirir conhecimentos sobre as definições, classificações e diferenças causadas pela deficiência, este trabalho tem como objetivo apresentar esclarecimentos teóricos e práticos sobre paralisia cerebral. Para tanto, organizou-se um texto explicativo dos principais motivos que causam as alterações na postura e movimento das crianças com deficiência, pois é sabido que o conhecimento dos pais pode interferir no cuidado e na educação, considerando que os pais desempenham um papel essencial como educadores de seus filhos, especialmente durante os primeiros anos de formação, e que isso proporcionará a todos oportunidades de participação, aprendizado e crescimento.

Palavras-chave: Paralisia cerebral. Atividade motora. Relações familiares.

\section{ABSTRACT}

One aspect that cannot be ignored is the impact of cerebral palsy in the internal dynamics of the family, due to the fact that its members are confronted with a different reality which requires adaptations and changes daily. The way parents, caregivers and family members adjust is crucial for the future of the child and of the people involved. Due to the difficulties of parents and caregivers of children with cerebral palsy in acquiring knowledge about the definitions, classifications and differences caused by disability, this work aims to present theoretical and practical explanations about cerebral palsy. Therefore, an explanatory text was organized with the main reasons that cause changes in posture and movement of children with disabilities, because it is known that the knowledge of parents can interfere in the care and education, whereas parents play an essential role as educators of their children, especially during the early formative years, and that this will provide all opportunities for participation, learning and growth.

Key words: Cerebral palsy. Motor activity. Family relations.

\footnotetext{
* Professor doutor do curso de Ciências da Atividade Física na área de Atividade Física e Esporte Adaptado e Comportamento Motor da Escola de Artes, Ciências e Humanidades da Universidade de São Paulo (EACH-USP) - Av. Arlindo Béttio, I000 - Ermelino Matarazzo - São Paulo - SP - 03828-000 - e-mail: carlosmonteiro@usp.br.** Fisioterapeuta, participante da organização e implementação de Atividade Física e Esporte Adaptado na EACH-USP, membro do Grupo de Estudos e Pesquisas em Capacidades e Habilidades Motoras (GEPCHAM).
} 


\section{INTRODUÇÃO}

Existem diferentes definições para paralisia cerebral $[14,7,3]$, mas atualmente é importante citar o trabalho de Bax [4] e a revisão deste por Rosenbaum [I7], que apresentaram a seguinte definição: "Paralisia cerebral é um grupo de desordem permanente do desenvolvimento da postura e movimento, causando limitação em atividades, que são atribuídas a um distúrbio não progressivo que ocorre no desenvolvimento encefálico fetal ou na infância. A desordem motora na paralisia cerebral é frequentemente acompanhada por distúrbios de sensação, percepção, cognição, comunicação e comportamental, por epilepsia e por problemas musculoesqueléticos secundários" [2, IO, I5, I8].

Percebe-se pela definição que a paralisia cerebral é uma entidade clínica complexa, com níveis de alta incidência, vários níveis de gravidade e complicações diversas, muitas vezes necessitando de cuidados especiais. Porém, os pais e cuidadores de indivíduos com paralisia cerebral relatam muita dificuldade em conceituar a doença e compreender suas causas e repercussões. Esta dificuldade em compreender as características e dificuldades da criança causa um impacto negativo na família e nos cuidadores, aumenta os questionamentos, gera angústia e ansiedade [I6]. O conhecimento limitado e uma visão pessimista quanto à potencialidade da criança repercute na dinâmica familiar e diminui os investimentos no tratamento e na educação [I6].

Desta forma, é fundamental o desenvolvimento de projetos que possibilitem informar pais e cuidadores de crianças sobre as principais características da paralisia cerebral, viabilizando uma fundamentação teórica e prática que possibilite a interpretação da paralisia cerebral pela família.

\section{OBJETIVO}

O objetivo deste trabalho é diminuir a insegurança e a falta de conhecimento por parte dos pais e cuidadores de crianças com paralisia cerebral, oferecendo uma fundamentação teórica prática de forma coerente e simplificada.

\section{MÉTODO}

Para tanto, optou-se em desenvolver um texto explicativo para pais e cuidadores de crianças com paralisia cerebral que apresente a definição, classificação, diferença na realização de movimento e características motoras. O texto completo organizado para pais e cuidadores será apresentado nos resultados.

\section{RESULTADOS}

É difícil falar em paralisia cerebral sem explicar como funciona o desenvolvimento motor normal do ser humano. Após o nascimento, a criança se movimenta, principalmente, devido a atos involuntários chamados de reflexo, que pode ser definido como: "Ato ou fenômeno, motor ou secretor, desencadeado pelo sistema nervoso fora da intervenção da vontade, em resposta a uma estimulação das terminações nervosas sensitivas" [9]. Conforme a criança adquire novas vivências, como o conhecimento do próprio corpo e a integração deste com o meio ambiente, seu sistema nervoso como um todo, inclusive o cérebro, é estimulado e evolui. Desta forma, a criança começa a controlar seus movimentos e, ao invés de realizar, na maioria, atos involuntários, inicia atos voluntários e atos motores automáticos, ou seja, respostas motoras mais refinadas e que necessitam de vivências anteriores.

A função do cérebro, a respeito da nossa conduta motora, é fundamentalmente a de receber, interpretar os estímulos sensoriais e nos oferecer a habilidade de nos movimentar e realizar atividades específicas, mantendo sempre uma postura e equilíbrio que nos possibilite executar tarefas especializadas [I2].

Cada movimento que fazemos é o resultado de um acúmulo de informações sensoriais e motoras que o cérebro adquiriu durante sua fase de maturação e das modificações que ocorrem devido a aprendizagens diárias. Todas essas informações recebidas são interpretadas e armazenadas, estando prontas para serem usadas quando necessário.

Nós andamos, corremos e executamos diferentes tipos de movimentos; no entanto, quando nos deparamos com uma movimentação diferente, percebemos a dificuldade que temos de nos adaptarmos. Por exemplo: quem já tentou iniciar um curso de dança de salão ou uma ginástica aeróbica? São todos 
movimentos e ritmos diferentes dos que empregamos em nosso dia a dia. Nosso cérebro não está acostumado; por isso, precisamos de várias repetições e tempo para poder nos adaptar a essas novas atividades.

Precisamos nos concentrar passo a passo para depois juntarmos os movimentos e executá-los perfeitamente. O mais interessante é que, apesar do movimento ser igual, cada um irá realizá-lo de uma forma diferenciada. Esta individualidade ocorre devido a diferentes vivências que cada um de nós tem durante a vida e a forma como nosso cérebro interpreta determinado estímulo. O mais importante é que podemos aprender novos movimentos e modificá-los durante qualquer fase da vida.

Vamos analisar o que ocorre nas crianças com paralisia cerebral. Existem casos de bebês que, ao nascerem, não conseguem nem mesmo se alimentar, e, nesses casos, é mais fácil o diagnóstico. No entanto, vários recém-nascidos com paralisia cerebral apresentam os mesmos reflexos que qualquer criança; por isso, conseguem mamar e movimentar-se involuntariamente. Assim, passam por todas as avaliações médicas sem revelar problemas que irão se apresentar mais tarde, quando o bebê começa a reagir aos estímulos impostos pelo meio ambiente, vivenciar seu corpo no espaço e se movimentar automaticamente [II, 8]. Esses controles automáticos são todos realizados pelo cérebro, que por algum motivo foi lesado na paralisia cerebral e não responde normalmente aos estímulos oferecidos.

Esta dificuldade de movimento que a criança começa a apresentar influenciará na perda de oportunidades de vivenciar posições diferentes e variedades de movimentos, o que irá representar um atraso na sua maturação cerebral e com certeza uma maior dificuldade em seu desenvolvimento sensorial e motor futuramente.

No desenvolvimento da criança com paralisia cerebral, é necessária uma quantidade e variedade de estímulos adequados para ajudar a criança a desenvolver todas as suas potencialidades, não só estímulos físicos, mas também cognitivos. Acredita-se, cada vez mais, que tanto o corpo como a mente devem ser trabalhados em harmonia, para que possamos oferecer à criança possibilidades de atingir todo o seu potencial. Diante disso, percebe-se a necessidade de uma equipe multidisciplinar onde fisioterapeutas, terapeutas ocupacionais, fonoaudiólogos, pedagogos especializados, psicólogos e vários outros profissionais envolvidos oferecerão à criança possibilidades de conhecimentos e movimentos que ela não conseguiria realizar sozinha.

\section{COMO OCORRE O MOVIMENTO}

Quando falamos em estimular uma criança com paralisia cerebral, estamos objetivando melhorar sua postura e movimento. Mas como o cérebro controla os movimentos?

O movimento ocorre por dois fatores importantes: a existência dos ossos, que são responsáveis pela sustentação do corpo; e os músculos, que se responsabilizam pelo movimento desses ossos e, consequentemente, pelos nossos movimentos. O que mais nos interessa quando falamos em movimento é analisar como o cérebro controla essa ação muscular.

O cérebro não entende nada de músculos, mas sim, de movimento. Quando aprendemos um determinado movimento, seja quando bebê ou adulto, o cérebro armazena o ato motor propriamente dito, independente de qual músculo deve ser contraído. Mesmo voluntariamente, nós não conseguimos contrair apenas um músculo; todas as ordens do cérebro serão feitas pensando no movimento. Ao tentar mover apenas um dedo, com certeza você estará contraindo músculos do braço, ombro e várias partes do corpo que você nem imagina participar do movimento pretendido, tudo isso para poder executar a ação, teoricamente simples, de mover um dedo.

Desta forma, não há nenhum músculo no corpo que possamos colocar em ação separada e independente do efeito colateral de outros. Não podemos fazer isso nem voluntária nem involuntariamente.

Quando você está fazendo uma atividade física - por exemplo, um exercício abdominal - os músculos das pernas, braços e outros também estão contraídos para estabilizar o movimento; ao mesmo tempo, os músculos das costas estão relaxados para não se oporem ao movimento e permitirem a execução do abdominal. Nem você e nem mesmo o cérebro sozinho conseguem controlar individualmente os músculos; no entanto, conseguem realizar perfeitamente o movimento.

Imagine quantas adaptações são necessárias para um simples movimento de andar! Todos os músculos participam simultaneamente e em perfeita harmonia, contraindo em algumas vezes e relaxando a seguir, pois qualquer falha significa uma desestruturação do movimento. Podemos concluir, após estas observações, que os músculos individuais perdem sua identidade, sendo que aumenta a importância de um trabalho muscular em conjunto. 
Para o ser humano se movimentar, de tantas formas variadas e complexas, existe um mecanismo automático chamado de mecanismo postural normal.

Este mecanismo, que nos oferece pré-requisitos para uma atividade funcional normal, é influenciado e depende da harmonia de dois fatores: o tônus postural normal e a inervação recíproca.

O tônus postural normal é um estado de semicontração da musculatura. Mesmo quando o músculo está em repouso, certa quantidade de tensão permanece, É um estado de alerta do músculo. Este grau residual de contração do músculo esquelético denomina-se tônus muscular. Tônus [9] é o estado de tensão permanente e involuntário dos tecidos vivos, especialmente, do tecido muscular esquelético sob a dependência do Sistema Nervoso Central (SNG) e Sistema Nervoso Periférico (SNP).

O tônus está diretamente relacionado com o movimento, pois o aumento e a diminuição do tônus de um determinado músculo irão influenciar diretamente na movimentação. O tônus muscular normal é difícil de ser medido, pois varia de pessoa para pessoa e até mesmo de momento para momento. Assim, em uma pessoa nós podemos, durante o dia, observar um tônus muscular mais elevado, dependendo do seu estado de alerta, preocupação e o próprio stress, que é tão estudado hoje em dia.

Podemos, ainda, observar um tônus muscular mais baixo, por esta pessoa estar mais relaxada e despreocupada. Todos esses são fatores que podem influenciar no nosso tônus muscular. $\mathrm{O}$ importante não é ter um tônus mais alto ou mais baixo, e sim um tônus normal, que nos propicie condições de controlar nossos movimentos coordenadamente. Podemos classificar um tônus normal como aquele que é alto o suficiente para vencer a ação da gravidade e baixo o suficiente para não se opor ao movimento pretendido. Ou seja, se alguém tiver um tônus muito baixo, não terá força suficiente para vencer a gravidade e, como consequência, não conseguirá mover-se por falta de tonicidade muscular. Por outro lado, se tiver o tônus muito alto, as articulações desses músculos alterados ficarão tão fixas que não conseguirão executar o movimento.

É importante ressaltar que o cérebro é que controla em que momento este ou aquele grupo muscular deve estar com o tônus mais alto ou mais baixo para uma harmonia nos movimentos.

No caso da paralisia cerebral, um dos maiores problemas é justamente o tônus muscular. Isto quer dizer que, por algum motivo, o cérebro não consegue controlar a contração dos músculos envolvidos em um determinado movimento ou mesmo em uma determinada postura.

Sem um controle organizado do cérebro, este tônus pode variar de alto para baixo. Alguns têm o tônus tão baixo (hipotônicos) que não conseguem vencer a gravidade em nenhum movimento, nem mesmo para levantar a cabeça $[6,8]$. Outros têm o tônus tão alto (hipertônicos ou espásticos) que não conseguem realizar nenhum movimento, pois um músculo não relaxa para permitir a contração de outro e/ou músculos que exercem funções contrárias em um determinado movimento se contraem ao mesmo tempo, não ocorrendo movimento algum [5, 9, I2].

A inervação recíproca é outro mecanismo importante para uma atividade funcional normal. Apesar do nome complicado, isso nada mais é do que uma perfeita integração entre todos os músculos do corpo quando executamos um movimento. $O$ cérebro tem o perfeito controle de qual músculo deve ser relaxado, e qual deve ser contraído para que o movimento ocorra com perfeição.

É justamente o exemplo do exercício abdominal. Assim que realizamos um exercício, o cérebro tem que controlar qual grupo muscular contrai, qual relaxa e quais irão auxiliar o movimento. E o mais importante, qual a intensidade de participação de cada grupo muscular. Sem este controle, o movimento não será harmonioso.

Esta interação deve ocorrer em qualquer tipo de movimento, seja com amplitude grande ou pequena, seja um movimento próximo do tronco, como elevar os braços, ou movimento distante do tronco, como os de abrir e fechar as mãos. Imagine o cérebro controlando todos os músculos das mãos, dedos, braços, ombros e todos os músculos do corpo que são necessários para executar tarefas como tricotar, usar uma tesoura ou até cumprimentar outra pessoa.

Além dos problemas de tônus muscular, na paralisia cerebral também ocorre a falta de inervação recíproca. Se o cérebro estiver lesado, como cada músculo será informado para se contrair ou relaxar para que possa ou não ocorrer determinado movimento? Ou melhor, em qual momento um músculo que flexiona deve relaxar para que o outro estenda e, desta forma, ocorra o movimento harmônico? Devido à lesão cerebral, 
essa coordenação central não existe ou pelo menos está desordenada. É justamente por isso que surgem os movimentos anormais. A intenção e a vontade do movimento existem, mas a perfeita coordenação para que surja um movimento produtivo não ocorre [I].

Percebemos isso quando observamos uma criança com paralisia cerebral se movimentar. A intenção do movimento existe, ela quer rolar, sentar e andar. No entanto, o cérebro, que é o responsável por coordenar e organizar esses movimentos, está alterado. Assim, ao tentar executar um movimento qualquer, não existirá controle dos músculos, aparecendo um movimento com menos coordenação e funcionalidade [4, I7].

As dificuldades motoras apresentadas serão definidas dependendo de quanto, como e onde o cérebro foi afetado. Cada criança com paralisia cerebral apresentará um problema motor específico e diferente dos outros, com seus limites e potenciais individuais.

\section{DISCUSSÃO}

Mesmo com o avanço da Tecnologia e da Medicina, os pais e cuidadores de crianças com paralisia cerebral ainda têm pouco acesso às informações como definição, quadro clínico e evolução do estado de saúde. Este conhecimento limitado leva os pais a terem uma visão pessimista sobre o potencial de seus filhos, dificultando também a transferência das habilidades adquiridas nas sessões de reabilitação para o dia a dia das crianças.

Pouco se tem descrito sobre paralisia cerebral voltada para pais e cuidadores. Porém, o esclarecimento sobre a deficiência é responsabilidade dos profissionais envolvidos no processo de habilitação e que têm contato direto com os pais. Ribeiro [I6] descreve que os profissionais da saúde admitem oferecer poucas informações aos pacientes com deficiência e seus familiares. Entre os itens mencionados como insuficientes, estão: necessidade de discutir os questionamentos; promover troca de experiências entre as famílias; e oferecer informações em relação às preocupações dos pais com o cuidado de seus filhos.

Segundo Prudente [I5], um aspecto que não pode ser ignorado é o impacto da paralisia cerebral na dinâmica interna da família, devido ao fato de que seus membros são confrontados com uma realidade diferente que exige adaptações e modificações diárias. A forma como os pais, cuidadores e membros da família se ajustam é crucial para o futuro da criança e das pessoas envolvidas diretamente. Cada família é única e irá processar esta experiência de maneiras diferentes. Muitas famílias são sobrecarregadas, considerando aspectos finaceiros e emocionais, o que pode causar uma ruptura familiar; por outro lado, existem famílias que são fortalecidas, e a criança deficiente agrega fatores de união. Corroborando com esta afirmativa, Lima [I3] mostra a importância do envolvimento familiar no processo de habilitação, citando que os profissionais que assistem a criança desempenham papel fundamental na transformação do olhar dos pais em relação ao filho. Considera ainda que esse olhar socialmente moldado pela desinformação, preconceito e carga histórica negativa pode ser transformado com o conhecimento e o apoio adequado.

Enfatizando a importância para pais e cuidadores de crianças com paralisia cerebral conhecerem as características da deficiência para a melhora do desenvolvimento das crianças, Ribeiro [I6] afirma que os pais de crianças que não apresentam nenhuma alteração motora e que possuem melhor conhecimento em relação às etapas da evolução da criança, por meio de revistas e livros disponíveis, conseguem executar com mais eficiência as práticas de cuidado com o filho, e isto interfere de forma positiva no desenvolvimento da criança. Pode-se supor, então, que o conhecimento dos pais de crianças com deficiência também pode interferir no cuidado e na educação prestados a elas, considerando que os pais desempenham um papel essencial como educadores de seus filhos, especialmente durante os primeiros anos de formação, e que isso proporcionará a todos oportunidades de participação, aprendizado e crescimento.

Ribeiro [I6] também mostra que a insatisfação dos familiares em relação às informações recebidas da equipe de saúde (médico, fisioterapeuta, fonoaudiólogo e terapeuta ocupacional) inclui: desejo ser esclarecido sobre as opções de tratamento; ter informações a respeito dos progressos da criança; obter informações a respeito da condição de deficiência da criança (causas, evolução e previsões).

\section{CONCLUSÃO}

Por todos os fatores apresentados, concluímos que o pouco conhecimento a respeito da paralisia cerebral influencia negativamente o processo de 
orientação familiar, dificultando a intervenção e diminuindo a adesão no processo de habilitação adequado. Consideramos este artigo interessante para os pais e cuidadores de crianças com paralisia cerebral, de modo a eliminar dúvidas sobre a definição da deficiência e para que se tornem mais seguros e envolvidos, permitindolhes dar maior assistência ao filho, aderir ao tratamento fornecido pelos profissionais de saúde e transferir o aprendizado à vida cotidiana.

\section{REFERÊNCIAS BIBLIOGRÁFICAS}

[I] AICARDI, J.; BAX, M. Cerebral palsy. In: AICARDI, J. Diseases of the nervous system in childhood. London: Mac Keith Press, 1992, p. 330-374. (Clinic in developmental medicine).

[2] AMATYA, B.; KHAN, F. Rehabilitation for cerebral palsy: Analysis of the Australian rehabilitation outcome dataset. Journal of Neurosciences in Rural Practice, v. 2, n. I, p. 43-49, 20 II.

[3] AMERICAN ACADEMY FOR GEREBRAL PALSY (AACPDM). What is Cerebral Palsy? Milwaukee: AACPDM, [c20II]. Disponível em: <http://www.aacpdm.org $>$. Acesso sem data.

[4] BAX, M. et al. Proposed definition and classification of cerebral palsy. Developmental Medicine \& Child Neurology, v. 47, p. 57I-576, 2005.

[5] CASALIS, M. E. P. Espasticidade: cinesioterapia e terapia medicamentosa. In: Reabilitar, n. 4, p. III5, 1999 .

[6] CAMPBELL, W. W. De Jong: o exame neurológico, 6. ed. Rio de Janeiro: Guanabara \& Koogan, 2007.

[7] DIAMENT, A.; GYPEL, S. Neurologia infantil, 4. ed. São Paulo: Atheneu, 2005.

[8] FOnSECA, L. F.; PIANETTI, G.; XAVIER, G. C. Compêndio de neurologia infantil, I. ed. São Paulo: Ed. Medsi, 2002.

[9] GARNIER, M.; DELAMARE, V. Dicionário de termos técnicos de medicina, 20. ed. São Paulo: Organização Andrei, I984.

[IO] GORTER, J. W. et al. The relationship between spasticity in young children (I8 months of age) with cerebral palsy and their gross motor function development. BMC Musculoskeletal Disorders, v. IO, 2009, 9 p.

[II] JAN, M. M. S. Cerebral palsy: comprehensive review and update. Annals of Saudi Medicine, v. 26, n. 2, p. I23-132, 2006.

[12] KANDEL, E. R.; Schwartz, J. H.; Jessell, T. M. Fundamentos da neurociência e do comportamento. Rio de Janeiro: Prentice-Hall do Brasil, 1997.

[i3] LIMA, R. A. B. C. Envolvimento materno no tratamento fisioterapêutico de crianças portadoras de deficiência: compreendendo dificuldades e facilitadores, 2006, I44f. Dissertação (Mestrado em Ciências da Saúde) - Faculdade de Medicina da Universidade Federal de Minas Gerais, Belo Horizonte, 2006.

[I4] Little club clinics in developmental medicine. Child neurology and cerebral palsy: a report of an international study group. London: William Heinemann Medical Books, I960. (Little club clinics in developmental medicine series, v. 2).

[I5] PRUDENTE, G. O.; BARBOSA, M. A.; PORTO, C. C. Relation between quality of life of mothers of children with cerebral palsy and the children's motor functioning, after ten months of rehabilitation. Revista Latino-Americana de Enfermagem, vol. I8, n. 2, p. 149-55, 2010.

[i6] RIBEIRO, M. F.; BARBOSA, M. A.; PORTO, G. C. Paralisia cerebral e síndrome de Down: nível de conhecimento e informação dos pais. Ciência \& Saúde Coletiva, v. I6, n. 4, p. 2099-2106, 2011.

[i7] ROSENBAUM, P. et al. A report: the definition and classification of cerebral palsy. Developmental Medicine \& Child Neurology, v. 49, supl. Io9, p. 8-I4. 2007.

[I8] SORSDAHL, A. B. et al. Change in basic motor abilities, quality of movement and everyday activities following intensive, goal-directed, activity-focused physiotherapy in a group setting for children with cerebral palsy. BMC Pediatrics, v. IO, n. 26, 20IO, II p. 